Cambouis, la revue des sciences sociales aux mains sales

\title{
Idéal de l'individu, individu statistique, individu social
}

\section{Remarques sur l'individuation des pratiques et des « opinions » dans la méthodologie du questionnaire}

\section{Michel Grumbach}

Université de Paris VIII

Résumé: La notion de Personne constitue, depuis environ deux siècles, le foyer du système de valeurs officielles des sociétés occidentales. Mais est-il possible pour autant, comme l'écrit Marcel Mauss, de considérer que la « révolution des mentalités est faite » et que cette notion, à la fois catégorie de l'idéologie et concept sociologique, décrit adéquatement les réalités sociales de l'existence individuelle ? Et, au-delà de Marcel Mauss et des sociologues, la statistique, au moins dans certains de ses actes méthodologiques, ne partage-t-elle pas le «trésor commun » de cette même conviction ? La méthodologie établie du questionnaire standardisé ne tient-elle pas qu'il n'y a d'acteur et d'activité dignes de ce nom qu'individuels ? Qu'un interviewé ne peut que répondre pour lui-même et en son nom propre ? Et qu'il est toujours « équipé » symboliquement pour le faire ? Cette communication se propose d'envisager l'éventualité, et d'analyser les effets, d'une telle rencontre entre les valeurs de la Personne et la méthodologie du questionnaire statistique.

Date de publication : $8 / 7 / 2021$

Dossier : Sociologie et statistique

Comment citer : $10.52983 /$ crev.vi0.49

Licence:Cambouis publie ses contenus selon les termes de la Licence Creative Commons Attribution - Pas d'Utilisation Commerciale - Pas de Modification 4.0 International. Les auteurices gardent leurs droits de propriété intellectuelle pleine et entière sur leurs articles. 


\section{Idéal de l'individu, individu statistique, individu social}

\section{Remarques sur l'individuation des pratiques et des « opinions » dans la méthodologie du questionnaire}

\section{Michel Grumbach}

Université de Paris VIII

« Depuis ce temps, la révolution des mentalités est faite, nous avons chacun notre "moi", écho des Déclarations des Droits, qui avaient précédé Kant et Fichte. » Marcel Mauss, Une catégorie de l'Esprit Humain : la notion de Personne, celle de «Moi»

La notion de Personne constitue, depuis environ deux siècles, le foyer du système de valeurs officielles des sociétés occidentales. Mais est-il possible pour autant, comme l'écrit Marcel Mauss, de considérer que la « révolution des mentalités est faite » et que cette notion, à la fois catégorie de l'idéologie et concept sociologique, décrit adéquatement les réalités sociales de l'existence individuelle ? Et, au-delà de Marcel Mauss et des sociologues, la statistique, au moins dans certains de ses actes méthodologiques, ne partage-t-elle pas le «trésor commun » de cette même conviction ? La méthodologie établie du questionnaire standardisé ne tient-elle pas qu'il n'y a d'acteur et d'activité dignes de ce nom qu'individuels ? Qu'un interviewé ne peut que répondre pour lui-même et en son nom propre ? Et qu'il est toujours « équipé » symboliquement pour le faire ? Cette communication se propose d'envisager l'éventualité, et d'analyser les effets, d'une telle rencontre entre les valeurs de la Personne et la méthodologie du questionnaire statistique.

\section{L'individu comme unité statistique}

Au-delà de la rigueur mathématique de sa définition formelle comme élément d'un ensemble, la notion d'unité statistique, lorsqu'elle désigne la personne physique, ne laisse-t-elle pas apercevoir certains traits classiquement associés à la catégorie éthico-psychologique de la Personne : distinction, permanence, unité, autonomie, indépendance ? C'est dans la méthodologie du recueil de l'information (questionnaire, interview) que se lit le mieux la vraie nature de l'individu, lorsque, dépouillé de son habit mathématique, il endosse la tenue banale de l'interviewé. C'est sans doute qu'il est impossible de fabriquer un 
questionnaire et de réaliser une interview sans engager, tant dans la manière d'interroger que dans ce à propos de quoi on interroge, un minimum de présupposés relatifs à ce qu'est un être humain.

\section{L'individu selon la procédure de questionnement}

L'observation statistique par questionnaire repose sur la délégation de l'exécution à l'observé. La légitimité scientifique d'une telle délégation suppose entre autres une condition particulière : que tous les interviewés dans leurs réponses soient susceptibles de faire, aussi spontanément, le même partage entre ce qui leur est propre ou personnel et ce qui ne l'est pas. Ou encore que puisse toujours être exactement connu le titre de l'effectuation, c'est-à-dire le titre (disons, en gros, personnel ou non) auquel l'interviewé était engagé dans l'activité ou l' « opinion » (goût, préférence...) qu'il rapporte. Il s'agit là d'une condition sine qua non de validité statistique et interprétative. De validité statistique, parce que cette propriété des réponses constitue un aspect de leur comparabilité et donc de la possibilité de leur agrégation. De validité interprétative : si ce titre est ignoré, l'interprétation de la relation entre l'activité rapportée et la variable indépendante retenue devient aveugle.

Un exemple imaginaire : soit les emprunts de livres dans une bibliothèque municipale. Supposons qu'on veuille analyser selon le sexe soit l'emprunt luimême, soit les livres empruntés. Si on ignore à quel titre, personnel ou non, ces emprunts sont effectués, comment est-il possible :

- d'agréger les réponses féminines ou masculines (comparabilité intra-sexe des réponses);

- de comparer les réponses féminines et masculines, si d'aventure il se révélait que les femmes mariées n'empruntent que pour le ou les autres membres de la famille et les hommes, mariés ou non, pour eux-mêmes (comparabilité inter-sexe des réponses);

- d'imputer l'emprunt ou les goûts au sexe et de les interpréter comme significatifs de l'activité ou du goût féminin?

Cette condition particulière de validité est-elle remplie dans le questionnaire statistique? Sa standardisation, telle qu'elle est conçue, l'assure-t-elle?

Disons tout de suite que dans la méthodologie habituelle cette question ne se pose pas parce qu'elle se donne, sous la figure de l'interviewé, une nature psychologique-type caractérisée justement par sa propension, naturelle ou sociale peu importe, à agir, « opiner » et répondre exclusivement à titre personnel.

En effet, on ne peut s'expliquer autrement, d'une part, la conviction que pour produire des réponses comparables il faut et il suffit d'interroger tout le monde de la même manière (standardisation de la procédure de questionnement) et, d'autre part, l'ignorance avérée du souci de connaître le titre de l'effectuation. Si on peut s'en passer, comme en témoigne en général l'absence dans le questionnaire d'un dispositif de questions ad hoc permettant de l'établir, cela ne peut être que parce qu'on s'est donné a priori chez tous les interviewés, à titre de propriété d'essence, l'action ou l'« opinion » à titre personnel et la réponse conforme, excluant ainsi en droit que ce problème soit posé. Le type de questionnement ayant cours se caractérise par le présupposé d'un « rapport au propre » identiquement constitué, également réalisé et aussi spontanément exprimé chez tous les interviewés ; il ne repose que sur le donné d'un individu qui, dans ses actes comme dans ses réponses, ne se donne toujours que lui-même. La « personne distincte » de la statistique se double donc d'une personne réellement distincte, tant par ses pratiques matérielles que symboliques. 


\section{L'individu selon les questions qu'on ne lui pose pas}

L'analyse du contenu des questions confirme-t-elle les remarques précédentes?

L'examen d'un grand nombre de questionnaires convainc aisément que dans l'ensemble, pour leurs rédacteurs, l'individu-type est bel et bien un acteur solitaire doublé, cela va de soi, d'un individualiste. Quelles que soient les activités qui fournissent la matière du questionnaire, il semble en général que l'interviewé les accomplisse seul et à titre personnel ou pour lui-même. Pour le dire avec les mots de Erving Goffman, n’apparaissent dans les questionnaires que des « individus "seuls" » et jamais des « individus "avec" » (Goffman, 1973a, p. 33-41).

Ce qui autorise à le dire, c'est précisément le constat de cette absence systématique, ou de la présence essentiellement fortuite, de certaines questions déterminées relatives à des dimensions de l'effectuation qui peuvent pourtant être tenues pour significatives, comme celle de la compagnie (fréquemment considérée comme un aspect secondaire et extrinsèque et, comme tel, classée dans les « circonstances de la pratique ») et celle du titre de l'effectuation ${ }^{1}$. Cet « oubli », ou la conviction qu'il s'agit là, somme toute, d'aspects négligeables, ne peut s'expliquer que par la «prénotion » de l'individu. Si l'on ne se sent pas systématiquement et constamment tenu de contrôler ces dimensions de l'effectuation, c'est, bien sûr, qu'on a admis tacitement une sorte d'idéal de l'action comme action individuelle, autonome et « personnalisée », libre et responsable, donc accomplie essentiellement par soi et pour soi. Bref, si on ne se le demande pas, et si on ne le demande pas, c'est qu'on se l'est accordé et qu'on l'a accordé2.

\section{L'individu dans le tableau et son commentaire}

Point n'est besoin de développer à nouveau ces mêmes remarques, qui peuvent être étendues tant à la lecture des tableaux de contingence qu'à leur commentaire.

On devrait pouvoir nous accorder que, dans la lecture muette des tableaux, on préjuge fréquemment du caractère individuel des propriétés agrégées et également de la distribution homogène de ce même caractère selon les diverses valeurs d'une même variable et selon les diverses variables (voir l'exemple pris en 2). On lit spontanément les tableaux comme ont été construits les questionnaires.

Il suffit de parcourir les commentaires ou comptes rendus d'enquêtes pour s'apercevoir que l'individu constitue bien l'acteur ou le vecteur des activités analysées. Et cela avec les ruptures de ton que suscite l'irruption d'un personnage aussi abstrait qu'étrange dans ce qui se veut l'univers concret de la description. Comme lorsqu'on lit, par exemple, dans un énoncé qu'il faut dire

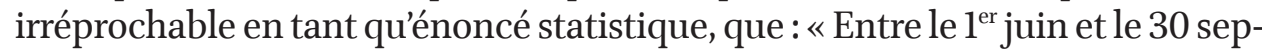
tembre 1969, 20460000 personnes distinctes sont parties en vacances, soit un taux de départ de 42,7 \% » (Le Roux, 1970, p. 68, c'est nous qui soulignons) ) $^{3}$.

Procédure et contenu du questionnement, lecture et commentaire de l'information obéissent à une logique commune définie par le postulat d'une égalité dans l'individuation et l'expression « personnalisée », et par une image de l'action et de l'acteur qui évoque l'idéal de la Personne. Sociologues et statisticiens ne connaissent, de ce point de vue, que des citoyens.
1 Pour ne rien dire de l'absence de questions relatives aux activités et « opinions » du conjoint qui est, in vivo, un peu plus que ce à quoi le réduisent habituellement les questionnaires : un statut, une profession, un diplôme. À ce sujet, il faut remarquer que la décontextualisation conjugale ou familiale qu'opère inévitablement le tirage d'échantillons composés d'individus prélevés aléatoirement interdit toujours de comparer les réponses des hommes et des femmes issus du même groupe familial et prive par là même de la possibilité de soupçonner le « réalisme » des réponses. Pour un problème en partie du même ordre, voir Bourdieu, Chamboredon et Passeron (1968, p. 66-67 et 243-240).

2 Pour un contre-exemple, voir Lemel (1974).

3 Sur ce problème du rapport entre énoncé statistique et énoncé sociologique, voir Passeron (1982) dans la présente publication. 


\section{Une illustration : les problèmes méthodologiques posés par quelques traits spécifiques des pratiques féminines}

On peut évaluer plus concrètement le degré d'adéquation descriptive de la notion d'individu telle que la postule l'usage établi du questionnaire statistique, lorsqu'elle s'impose comme catégorie organisatrice de l'observation, en analysant certains traits spécifiques des pratiques féminines ${ }^{4}$.

S'agissant des femmes mariées, les pratiques féminines posent d'une manière particulièrement pressante la question de savoir jusqu'à quel point il est possible et légitime de faire la part, dans l'imbroglio des réalités familiales, entre ce qui est personnel et ce qui ne l'est pas.

\section{Multiplicité et diversité des « délégations »}

La multiplicité et la diversité des « délégations » semblent être une caractéristique propre des activités des femmes, particulièrement lorsqu'elles sont inactives. Thorstein Veblen, qui y incluait, en plus du loisir et de la consommation, les pratiques religieuses, en fait l'essence même de la vie féminine, « vie délégataire » et « vie par procuration » (Veblen, 1970, p. 233-239). Préposées le plus souvent à l'approvisionnement alimentaire, elles peuvent aussi l'être à l'acquisition de biens de toute sorte. Énumérons dans le domaine des loisirs : achats de livres, disques, vidéocassettes et films ; inscription et emprunts en bibliothèque ; enregistrement des jeux aux courses ou au loto ; etc. Toutes ces délégations, lorsqu'elles ne sont pas connues comme telles, soulèvent le problème précédemment signalé des « croisements dangereux ». Peut-on dès lors comme on le fait habituellement s'en remettre à ces palliatifs que sont la familiarité, le bon sens ou la bonne volonté de l'interviewé pour corriger ce qui apparaît comme une lacune de la méthodologie du questionnement?

\section{Pratiques à double forme, personnelle et conjointe}

Les femmes au foyer sont, de par leur situation, particulièrement susceptibles d'exercer alternativement les mêmes activités, tantôt à titre effectivement personnel, tantôt conjointement avec leur époux. Et ce sans que l'observateur puisse savoir, s'il ne s'en est donné les moyens nécessaires, qui l'a emporté dans les réponses du «soi-je » ou du « soi-nous ». Des deux réponses possibles, celle qui renvoie à l'activité propre et celle qui renvoie à l'activité conjointe, comment préjuger que chez toutes et toujours l'usage propre l'emporte spontanément sur l'usage conjoint ou familial ? Ou l'inverse ? Cette situation pose typiquement le problème d'une connaissance minimale des logiques de réponse féminines, qui relèverait précisément d'une sociologie de l'individuation.

Un bon exemple en est le double usage de la télévision que font les femmes au foyer. Nettement distinctes dans le temps, ces deux activités diffèrent aussi par les conditions dans lesquelles elles se déroulent. Dans un cas l'épouse est seule (ou avec un ou plusieurs enfants en bas âge), elle s'organise selon un «plan d'activité personnelle » (Goffman) et dispose de la maîtrise du choix des programmes ; dans l'autre, elle est en compagnie du conjoint, presque toujours occupée (travaux ménagers, enfants, etc.) et sans maîtrise du programme. Ces deux usages peuvent, chacun à sa manière, s'analyser, selon les termes de Erving Goffman, comme des « adaptations secondaires » (Goffman, 1968), c'est-à-dire des prises de distance décisoires à l'égard de l'emprise de l'institution, par lesquelles le sujet préserve sa personnalité et
4 C'est en partie au schème éthique de la Personne que certaines recherches sociologiques « féministes » doivent d'avoir posé, par exemple, le problème sociologique et méthodologique de la place du genre dans la stratification sociale. «[...] si on doit considérer les femmes comme des personnes plutôt que comme des appendices des mâles, comment peut-on définir leur statut social en particulier lorsqu'elles sont inactives et ne peuvent donc être catégorisées sur la base de leur profession et de leur revenu propres? » (Acker, 1973, p. 941, c'est nous qui soulignons). Mais inversement, ce schème ne marque-t-il pas les limites de la clairvoyance possible en accordant sociologiquement et méthodologiquement aux femmes, trop vite et trop tôt, cela même qu'on énonce et qu'on dénonce comme leur étant refusé socialement dans le réel? 
signifie son autonomie. Ainsi, on peut voir dans les cumuls fréquents d'activités, généralement associés à l'écoute familiale, plus que l'expression d'une simple nécessité ou d'une inégalité : celle d'un détachement à l'égard de spectacles qu'on n'a pas choisis soi-même ${ }^{5}$. À l'inverse du temps que les femmes organisent elles-mêmes, pendant lequel elles peuvent insérer leur usage propre dans le flux des activités légitimes et déployer éventuellement leurs goûts personnels. Chassés-croisés de la dépendance féminine dans lesquels les femmes instituent et expriment leur autonomie tantôt par des moyens ou des activités légitimes (lecture, travaux divers, etc.) tantôt par une activité illégitime (télévision) parce que « privée », c'est-à-dire indûment soustraite au temps légitimement dû au travail familial. Des entretiens exploratoires conduits sur ce thème permettent de constater, sans qu'il soit encore possible d'en rendre compte, la diversité des rapports que les interviewées entretiennent à de telles situations, ainsi que celle des formes d'expression qu'ils peuvent prendre : « oubli » de l'usage propre, auquel porte l'» idéalisation » du rôle de la «bonne épouse »; « oubli » de l'usage conjoint ; oscillations et alternances $\mathrm{du}$ « moi-je » au « nous-on $»^{6}$; etc. Ces interviewées pourraient difficilement rentrer dans le cadre d'un questionnement standardisé qui postule la nature « représentative » des sujets.

On le voit, on ne peut ici, et à la différence de ce qu'il paraît souvent possible de faire dans le cas des hommes, s'en tenir à la devise commode $\mathrm{du}$ « one woman, one vote »! Puisqu'il y a non pas seulement deux réponses possibles mais réellement deux points de vue, deux rapports sous lesquels répondre, selon le degré auquel l'interviewé dispose et use ou non de ce «rapport au propre ». Il n'y a donc pas ici de « substances simples ». En effectuant, dans l'activité même, le partage clair du personnel et du conjugal, ces deux formes d'une même pratique permettent d'observer le rapport que les sujets entretiennent à cette situation et la manière dont ils l'expriment, c'està-dire d'analyser les conditions de constitution et les règles d'expression de ce qu'on nomme habituellement « avoir un point de vue personnel». Ce qui, par ailleurs, pourrait être aussi une approche, entre autres, de la domination dans le cadre du couple ou de la famille.

\section{Des pratiques exclusivement conjointes}

Enfin, il n'est pas rare qu'une série d'activités soient, pour certaines catégories de femmes, inaccessibles en dehors de la présence formelle de leur conjoint ou, au mieux, presque exclusivement exercées en sa compagnie. C'est fréquemment le cas de nombre d'activités de loisir, des épouses actives en particulier : cantonnement quasi exclusif à la télévision familiale; « sorties » diverses; etc. Si l'on n'y prend garde, ces réponses « collectives » peuvent fort bien être agrégées à des réponses effectivement personnelles et interprétées comme telles, c'est-à-dire considérées comme significatives de la variable indépendante sous le rapport de laquelle elles sont analysées. Ceci n’aboutit-il pas dans nombre de cas à compter deux fois la même réponse, une fois pour chaque sexe - quel que soit celui des deux conjoints qui est parvenu à imposer son choix — et par-là même à surestimer le « conformisme conjugal » au détriment d'éventuelles différences?

Le cas des pratiques exclusivement conjointes pose un problème difficile à trancher, dont voici un exemple : « Assez souvent l'épouse (en milieu populaire) accompagne son homme sur les lieux d'exercice de ses loisirs ; mais pour regarder, commenter, encourager. En ce sens, elle déclare ne pas exercer ces pratiques, et pourtant elle y prend part » (Lalive d'Epinay et al., 1982).

Devrait-elle dire qu'elle est allée à la pêche ? Ou qu'elle n'y est pas allée? Elle ne peut dire ni l'un ni l'autre. Que devrait-elle dire alors? Ce qu'on retrouve ici, c'est le revers obligé de la médaille individualiste. En effet, ce que montre
5 À propos de ces cumuls d'activités associées à l'écoute de la télévision familiale, voir Guilbert, Lowit et Creusen $(1965 ; 1967)$.

6 Pour un exemple des nombreuses remarques de ce type : « [...] l'information recueillie auprès d'un seul membre (du couple) risque d'être quelque peu biaisée en faveur ou en défaveur d'un conjoint. Il est malaisé de conjecturer dans quel sens l'information peut être distordue ; l'égocentrisme ou l'effacement de celui qui parle sont susceptibles de jouer dans des directions contraires » (Barbichon et Prado, 1982, p. 375-376, c'est nous qui soulignons). Voir également Guilbert, Lowit et Creusen $(1965 ; 1967)$. 
ce cas simple mais très répandu, puisque cette forme familiale des loisirs constitue une caractéristique distinctive du style de vie des classes populaires, c'est que le questionnaire, en sa méthodologie établie, est incapable de saisir autre chose que l'action individuelle et autonome, seule norme légitime de la pratique et de l'acteur. Relativement à une activité tenue et imposée comme principale, le co-pratiquant se trouve dès lors réduit au rôle, conçu comme purement passif, d'accompagnement et de « doublure ». Les exemples ne manquent pas qui jouent explicitement du couple du passif et de l'actif, essentiellement destiné à opposer tantôt le « populaire » au « cultivé », tantôt le féminin au masculin. Si les pratiques exclusivement conjointes posent un tel problème, c'est parce qu'on s'en tient toujours à la seule logique des unités individuelles. Ne faudrait-il pas admettre que « l'unité de participation » (Goffman) peut être également constituée par un collectif en tant que tel ?

Les remarques précédentes peuvent être étendues à l'appropriation et à l'usage des biens domestiques qui soulèvent les mêmes problèmes, et dont Erving Goffman a montré, à propos des objets personnels, l'importance qu'ils revêtent dans la constitution et le maintien de l'image de soi comme Individu ou Personne. Ici encore, le questionnement habituel, qui s'en remet intégralement à la spontanéité d'un interviewé idéal-typique crédité d'un sens inné de la propriété personnelle, aboutit le plus souvent, s'agissant des femmes mariées, à surestimer la part de l'ombre maritale (déclaration de non-possession, de non-usage, de non-pratique personnels) la mieux conçue et la plus énonçable parce que légitime, et à sous-estimer la part, plus ou moins illicite et auto-dissimulée mais réelle, de l'ego et du personnel.

\section{Retour à la méthodologie : individuation des réponses et observation statistique des propriétés « individuelles »}

Tentons de formuler de manière plus systématique les conséquences méthodologiques des remarques précédentes pour ce qui concerne l'observation statistique des diverses propriétés sociales.

Si on nous accorde, pour apprécier la pertinence méthodologique différentielle de l'individuation des réponses, relativement à une telle observation, de raisonner sur la base de la famille ou du couple conjugal, le problème est : compte tenu de la manière différente dont s'exercent les déterminations de classe ou de sexe, par exemple, sur les membres d'une même famille, l'individuation des réponses est-elle également et au même titre un réquisit de l'observation statistique des propriétés de classe et des propriétés de sexe?

Par rapport aux membres d'une même famille donnée, les déterminations de classe agissent dans une direction identique et suscitent une relative homogénéité sociale des individus qui la composent. Elles engendrent de l'identité. Dans ces conditions, l'individuation des réponses ne constitue pas une condition nécessaire de l'observation des propriétés de classe ; leur non-individuation n'est pas un obstacle. Inversement, les déterminations de sexe agissent, par définition, dans des directions opposées et suscitent de la différence et de la divergence. Ici, l'individuation des réponses est bien une condition sine qua non de l'observation statistique des propriétés de sexe et leur non-individuation une occultation. Autrement dit, du point de vue de l'observation des propriétés de classe, les membres d'une même famille sont approximativement substituables, et l'individuation n'est pas une propriété méthodologiquement pertinente des réponses. À l'inverse, parce que ces membres sont par définition insubstituables sous le rapport de l'observation des propriétés sociales de sexe, et plus généralement de toutes les détermina- 
tions qui, à l'intérieur de la famille, vont dans le sens de leur différenciation, l'individuation constitue bien une propriété méthodologiquement pertinente des réponses. L'observation statistique des propriétés de sexe suppose l'individuation des réponses.

Dès lors, si on refuse de tenir l'individuation symbolique pour une propriété essentielle et constitutive de l'individu social, et si on la suppose diversement constituée et plus ou moins exigible comme norme, cette condition de l'observation des différences de sexe apparaît inégalement remplie selon la place que les interviewés occupent dans les rapports susceptibles d'affecter de manière décisive cette propriété des réponses. Et la dépendance personnelle (principalement pour nous, sous sa forme conjugale ou familiale), à la différence de la domination de classe telle qu'elle s'exerce dans les sociétés industrielles, pourrait bien constituer un ensemble de rapports de ce type. En d'autres termes, si l'inégale individuation symbolique est un aspect de l'inégalité des sexes, alors les différences de sexe ne sont pas au même titre et aussi immédiatement saisissables ou données selon qu'il s'agit du sexe dominant ou du sexe dominé. Par exemple, parce qu'à l'unité tendancielle de point de vue et de langage caractéristique du dominant personnel dont les positions propres (intérêts, goûts, « opinions »...) se sont imposées avec succès comme position familiale légitime, pourraient s'opposer l'ambivalence, les « clivages », les oublis de soi et les oscillations variables du dominé pris entre une identification au groupe familial et une activité et un point de vue propres $^{7}$, entre loyalisme et dissidence.

Mais, alors, non données de manière immédiate, ces propriétés différentielles du sexe dominé ne pourraient être réellement « découvertes » qu'à la condition préalable d'avoir, et par décision de méthode, épuré et complété les phénomènes tels qu'ils se présentent spontanément, c'est-à-dire d'avoir, par des dispositions ad hoc, écarté certains types de réponse, ou bien, ce qui, dans les réponses, ne réfère pas en propre à l'interviewé lui-même, et ajouté les questions qui le contraignent à s'y rapporter. Il faut d'abord accepter de faire méthodologiquement la part d'un possible « conformisme conjugal », si l'on veut pouvoir le récuser sociologiquement.

Ainsi la prise en compte épistémologique et méthodologique de l'inégalité des sexes dès le questionnement pourrait bien être la condition même de la possibilité d'observer une telle inégalité. En ce sens, l'idée d'une sociologie différentielle de l'individuation, dont l'absence est suppléée grâce à « l'option par défaut » que constitue la catégorie éthico-psychologique d'individu, constituerait, dans «l'ordre des raisons », un préalable nécessaire à la sociologie de la famille et des rôles de sexe. Et ce n'est pas céder à l'» air du temps » que de reconnaître dans l'image culturelle de l'homme adulte ce qui définit implicitement le profil du questionnement dans l'enquête statistique. Au-delà, ces remarques ne prennent leur sens que par rapport au projet d'une sociologie qui se donnerait pour objet la construction sociale différentielle de la Personne.
$7 \quad$ Pour le problème partiellement analogue de l'identification du « stigmatisé », voir Goffman (1975, p. 127-148).

\section{L'hypothèse d'une sociologie différentielle de la construction sociale de la personne}

L'individu comme tel, pris comme objet sociologique ou comme profil de référence dans le questionnement, n’apparaît généralement que sous la forme d'un invariant social. Sorte de « moi » abstrait, universel et standard, sans histoire, sans sexe, sans état civil ni appartenance sociale. Et c'est la prégnance, à la fois philosophique et idéologico-politique, de cette catégorie de la Personne qui fixe des limites sociologiques à l'acquis que constitue la 
relativisation historique de l'individualité, chez Karl Marx (1957, p. 149-150) et Émile Durkheim $(1968$, p. 343-390 ; 1969; 1978), puis sa relativisation culturelle chez Marcel Mauss (1966).

Mais, s'il est manifestement impossible de tenir ce « culte de l'indivi$\mathrm{du} »($ Durkheim) pour une pure et simple chimère, n'est-il pas chimérique pour autant d'y voir une description « réaliste » de la réalité ? De tenir, sans autre forme de procès, tout individu pour un « foyer autonome d'activité » (Durkheim, 1969, p. 92-93) ? Si cela était, une sociologie différentielle de la construction sociale de la Personne deviendrait possible qui aurait pour projet d'étudier comment, par les différentes socialisations et resocialisations, sont socialement définis et réglés les divers usages et représentations de soi comme individu ; comment sont symboliquement structurés les divers types sociaux de « rapport au propre».

En dépit du postulat de l'invariance sociale du « moi » dont il est largement tributaire, Erving Goffman rencontre ce problème dans le cadre interactionniste qui est le sien ${ }^{8}$. En témoigne sa critique, dans Les relations en public, de l'inadéquation descriptive de la catégorie d'individu :

« Il ne faut pas oublier que - à un certain niveau du moins - les cadres sociaux et les manifestations sociales ne sont pas organisés en termes d'individus, mais en termes d'unités de participation. Certains endroits refusent les personnes seules, mais les reçoivent accompagnées ; d'autres endroits (certes, peu nombreux) pratiquent l'inverse. Certaines danses, interdites aux gens du même sexe, admettent des partenaires de sexes différents. Il y a des bars fermés aux femmes seules et aux groupes entièrement féminins, mais qui reçoivent les femmes accompagnées par des hommes. Les adolescents ne peuvent se promener dans certaines rues à certaines heures, sauf si ces malheureux sont accompagnés par des adultes. Enfin, il y a des heures et des lieux où les femmes ne trouvent pas convenable (ni avantageux) d'être seules. Pourtant, comme il est souvent vrai qu'un individu a qualité pour participer à chacune des deux unités, pour être seul ou pour être "avec", nous négligeons souvent le fait que les règles s'appliquent à deux niveaux : les unités admissibles et qui est admis dans l'unité. [...] Derrière ce souci [de préserver la réputation de certains établissements en en refusant l'accès aux femmes non accompagnées] se cache une question structurale. Puisque, d'après les critères conventionnels, les femmes, seules ou ensemble, constituent par elles-mêmes des unités de participation incomplètes, les hommes sont naturellement portés à percevoir ce manque. [...] Il est intéressant de remarquer que, dans le langage de tous les jours le terme de "femme seule" s'applique parfois à une femme qui entre en compagnie d'autres femmes, ce qui, à la lettre, est une erreur ; mais l'expression n'est pas ambiguë, car structuralement exacte. » (Goffman, 1973a, p. 36 ainsi que note 29, c'est nous qui soulignons) $)^{9}$.

Comme cette critique y invite, au nom de quoi accorder une égale et identique individuation matérielle et symbolique à tous les sujets sociaux, indépendamment de la diversité des conditions (capacités juridiques; dépendance ou « indépendance » économique; politique ; de circulation sociale et enfin cérémonielles du type de celles analysées par Erving Goffman) dans lesquelles ils se trouvent placés en raison, tout particulièrement, de leur appartenance de sexe et de classe d'âge ${ }^{10}$ ? Au nom de quoi accorder que certaines situations de dépendance personnelle justement — comme celle des jeunes inactifs, des épouses et des personnes âgées inactives - ne sont susceptibles d'affecter en rien les usages et les représentations de soi comme individu ? Si la Personne constitue un système de valeurs définissant un idéal de style de vie individuelle à prétention universelle, les différentes catégories de sexe ou d'âge peuvent y être très diversement préparées, autorisées à s'y conformer et susceptibles d'y adhérer. Par exemple, la plus grande proximité des valeurs et des modèles masculins à cet idéal suggère que seul peut-être le style de vie qu'il détermine
8 Voir, par exemple, en dépit d'incidentes destinées à signaler des écarts, tenant à l'appartenance de classe ou de sexe, par rapport au schéma standard de l'action qu'il analyse, la déclaration récurrente selon laquelle il étudierait un individu moyen des classes moyennes américaines (Goffman, 1973a, p. 337).

9 Voir également, pour cette même critique, Goffman (1973b, p. 79-83 et 145).

10 Stanley L. Payne rapporte le cas d'un recensement américain dans lequel la population fut sous-estimée parce que les questions étaient rédigées en termes de «personnes » et que les parents ne pensent pas aux enfants en très bas âge dans ces termes (Payne, 1951, p. 10). 
est apte à paraître le symboliser. Et encore, dans les limites biographiques socialement fixées, et avec les passages socialement obligés, qui définissent l'homme adulte et actif par opposition aux autres « âges » de la vie.

Cela ne revient-il pas à se demander si l'intérêt prédominant de la sociologie, depuis sa constitution, pour l'analyse de déterminations sociales, comme l'appartenance à une classe ou à un type, dont le « véritable individu » (Schumpeter, 1972, p. 168-169) est la famille et non la personne physique, n’a pas contribué à soustraire cette catégorie de l'individu à l'examen critique ? En effet, la préoccupation exclusive pour des propriétés sociales qui ne sont pas directement indexées, à la différence du sexe et de l'âge, sur des qualités propres des personnes physiques, dispense de s'interroger systématiquement sur les formes sociales de l'existence individuelle comme telle. Plus généralement, n'est-ce pas le projet constitutif de la sociologie, sous sa forme historiquement attestée, qui impliquait ce « sacrifice», dans la mesure même où, s'établissant contre tout individualisme, et tout dans l'individualisme, elle se donnait précisément pour tâche de « dépasser » des discours dont l'individu constituait le référent? Ne s'est-il pas conservé quelque chose dans ce « dépassement »? Et le bébé n'aurait-il pas été jeté avec l'eau du bain ? Si tel était bien le cas, il serait compréhensible que tant la réflexion actuelle sur les inégalités de sexe que l'orientation propre à l'interactionnisme contribuent à ce que soit reposé aujourd'hui ce problème de l'individu. 


\section{Bibliographie}

ACKER Jean, 1973, « Women and social stratification : a case of intellectual sexism », American journal of sociology, vol. 78, n 4, p. 936-945.

BARBICHON Guy et Prado Patrick, 1982, « Territoires de relations, territoires d'identité », Cahiers de l'OCS, vol. 8, p. 365-482.

Bourdieu Pierre, Chamboredon Jean-Claude et PAsseron Jean-Claude, 1968, Le métier de sociologue, Paris, Mouton.

Durkheim Émile, 1968, Les formes élémentaires de la vie religieuse, Paris, Presses Universitaires de France.

Durkheim Émile, 1969, Leçons de sociologie, Paris, Presses Universitaires de France.

Durkheim Émile, 1978, De la division du travail social, Paris, Presses Universitaires de France.

Goffman Erving, 1968, Asiles, Paris, Les Éditions de Minuit.

Goffman Erving, 1973a, Les relations en public, Paris, Les Éditions de Minuit.

GofFMAn Erving, 1973b, La présentation de soi, Paris, Les Éditions de Minuit.

Goffman Erving, 1975, Stigmate, Paris, Les Éditions de Minuit.

Guilbert Madeleine, Lowit Nicole et Creusen Joseph, 1965, « Problèmes de méthode pour une enquête de budgets-temps », Revue française de sociologie, vol. $6, \mathrm{n}^{\circ} 3$, p. 325-335.

Guilbert Madeleine, Lowit Nicole et Creusen Joseph, 1967, « Les budgets-temps et l'étude des horaires de la vie quotidienne », Revue française de sociologie, vol. 8, $\mathrm{n}^{\circ}$ 2, p. 169-183.

LALIVE D'ÉPINAY Christian, BASSAND Michel, Christe Étienne et Gros Dominique, 1982, « Persistance de la culture populaire dans les sociétés industrielles avancées », Revue française de sociologie, vol. 23, $\mathrm{n}^{\mathrm{o}} 1$, p. 87-109.

LE Roux Pierre, 1970, Les vacances d'été en 1969, Paris, Collections de l'INSEE, Série M, $\mathrm{n}^{\circ} 2$.

LEMEL Yannick, 1974, Les budgets-temps des citadins, Paris, Collections de l'INSEE, Série $\mathrm{M}, \mathrm{n}^{\circ} 33$.

MARX Karl, 1957, «Introduction à la critique de l'économie politique », in Contribution à la Critique de l'économie politique, Paris, Éditions Sociales.

Mauss Marcel, 1966, « Une catégorie de l'esprit humain : la notion de Personne, celle de "Moi" », in Sociologie et Anthropologie, Presses Universitaires de France.

PASSERon Jean-Claude, « Ce que dit un tableau et ce qu'on en dit », Communication au colloque Sociologie et statistique, Paris, INSEE/ SFS, 1982

PAYNe Stanley L., 1951, The art of asking questions, Princeton University Press.

SCHUMPETER Joseph, 1972, « Les classes sociales en milieu ethnique homogène », in Impérialisme et classes sociales, Paris, Les Éditions de Minuit.

Veblen Thorstein, 1970, Théorie de la classe de loisir, Paris, Gallimard. 